\title{
The research on Intelligent Monitoring System
}

\author{
Ying SHANG ${ }^{1, a}$, Jin-bo ZHANG ${ }^{2, b}$ \\ ${ }^{1}$ Shanxi Institute of International Trade \& Commerce ,Xi'an \\ ${ }^{2}$ Shanxi Institute of International Trade \& Commerce ,Xi'an \\ a81593866@qq.com,,526165466@qq.com
}

Key words: ZigBee technology, face recognition, intelligent monitoring, object tracking

Abstract. Aiming at the problem of inaccuracy of Face Recognition and No Object Tracking Ability with the current monitoring system, an intelligent monitoring system design scheme based on ZigBee technology is proposed, and the hardware and software module design of the system is implemented including data acquisition module, data transmission module and character face detection module, also the ZigBee technology networking principle and image compression and face recognition algorithm are expounded in this paper. The test results in the bank environment of the ZigBee technology based real-time monitoring system show that the proposed solution is simple, reliable and accurate with high transmission speed and the object tracking ability.

\section{Introduction}

Face recognition system ${ }^{[1]}$ taking face recognition technology as its core is today's high-tech research technology. Face recognition technology Combined with computer image processing technology and principle of biostatistics builds the mathematical model on the basis of biostatistics, uses the SVD-based feature extraction method to extract facial features from the video, and the prospect of the technology is huge and vast. To solve these problems, there is an urgent need to speed up the development of active prevention video surveillance system. The current real-time monitoring system can't identify facial features, and does not have the ability to track moving objects. In this paper, ZigBee based wireless network technique, face recognition method and image processing technology are introduced and analyzed, and the real-time intelligent monitoring system based on ZigBee is researched and designed ${ }^{[1]}$.

\section{The Overall Design of Intelligent Monitoring System}

The system is mainly composed of a data acquisition module, an object tracking module, a main controller, a face detection and recognition module, a wireless transmission module and a clock module, the overall solution is shown in Figure 1.

The facial features of a person is identified by a face detection module, The identification results are compared with the database files to identify or verify the identity of the person. The facial features of the target are extracted through the face detection module, and then compared with the database files, and if the verification fails, the alarm module will send out an alarm information to the system administrator; If the sensor detects a moving object, control commands will be sent so that the camera can track the object under the action of the stepping motor. Once the sensor detects a moving object, control commands generated according to the movement of the target will be sent by the object tracking module to a stepping motor so that the camera can keep the moving target under strict surveillance. An alarm will be reported when an object stays in this area for a specific 
length of time or exceeds the pre-defined time length of the clock module ${ }^{[2]}$.

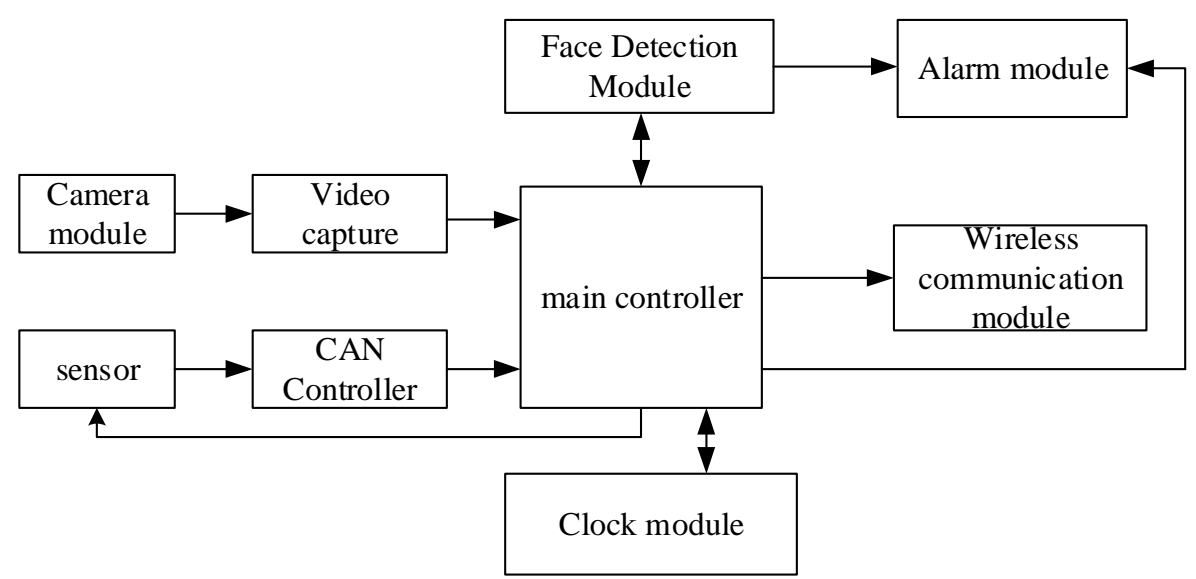

Fig.1 Design of Real-time Intelligent Monitoring System

\section{Hardware circuit design}

Face detection module ${ }^{[3,4]}$ uses DM6446 processor. DM6446 is a high-performance chip with strong data processing and object control capabilities for video and image processing. A programmable digital signal processor (DSP) core, an ARM processor and a video acceleration co-processor are integrated into the chip. The operating frequencies of the two integrated processor are respectively $594 \mathrm{M} \mathrm{Hz}$ and $297 \mathrm{MHz}$. DM6446 processor has a dedicated video processing subsystem which includes a front-end and an end output video interface. The video front-end input interface is used to receive the video data input from the external video decoder, and the video data processed by DM6446 through the end output video interface can be directly output to SDTV, LCD, HDTV and other display devices. DM6446 processor has a large storage capacity controller, External high-capacity hard disk can be connected through the DM6446 chip's memory controller. In addition, DM6446 processor also has a 64-channel EDMA controller, three serial ports, and three 64-bit general-purpose timers, and its power consumption is also very low ${ }^{[5]}$.

Video acquisition module ${ }^{[6]}$, Four-way video capture chip 7130 is used in video acquisition module. The chip uses H.264 / MPEG4 format for video compression, 100 frames per second refresh rate, four-channel total rate resources of 100 frames per second, maximum 24 road full real-time and Remote IE control are also supported. It also has a variety of other features, such as high-definition images, excellent compatibility, lightning protection circuit design, stable performance. Video acquisition block diagram is shown in figure 2. 


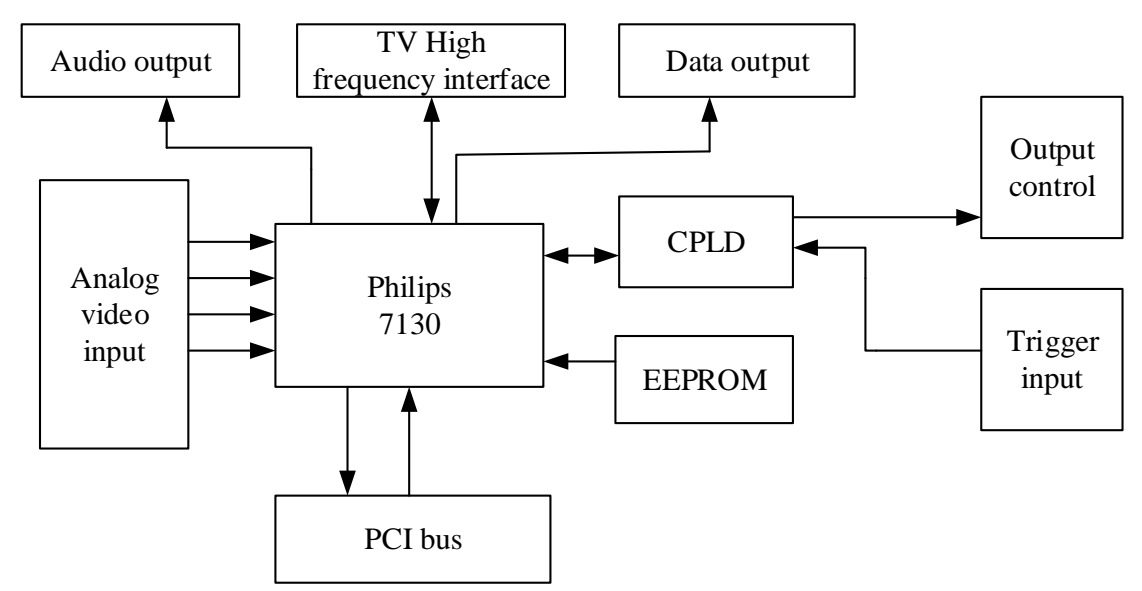

Fig.2 Video Capture Block Diagram

\section{Wireless communication module}

Wireless communication module using ZigBee wireless sensor network consists of sensors, routing and aggregation nodes. The main task of the module is transmitting the processed data.

ZigBee $^{[7]}$ is built on the IEEE802.15.4 standard. Its characteristics are as follows:

Low data transmission rate and low power consumption: In the low-power standby mode, two ordinary 5-cell batteries can be used for 6 to 24 months; Low cost, large network capacity: 65,000 devices can be accommodated; Short delay: Usually the delay is $15 \mathrm{~ms} \sim 30 \mathrm{~ms}$; Flexible frequency bands: Working frequency band including $2.4 \mathrm{GHz}, 868 \mathrm{MHz}$ (Europe) and $915 \mathrm{MHz}$ (US), all of these are free license band; Reliable transmission: compared with wi-fi or Bluetooch wireless network data transmission protocol, it is more suitable for monitoring and control industry.

The topology of ZigBee network mainly includes star-type network, cluster-type network and mesh-type network. Different network topologies correspond to different application areas. In addition, configurations for network nodes are also different in network topologies. The type of network node: Network coordinator、Full-function device and simplified function device.

\section{Zigbee technology}

In the system, the acquisition node and the sink node processor adopt the Zigbee technology chip cc2530. The CC2530 is a true system-on-chip solution for IEEE 802.15.4, Zigbee and RF4CE applications. It enables robust network nodes to be built with very low total bill-of-material costs. The C2530 combines the excellent performance of a leading RF transceiver with an industry-standard enhanced $8051 \mathrm{MCU}$, in-system programmable flash memory, 8-KB RAM, and many other powerful features. The CC2530 comes in four different flash versions: C2530F32/64/128/256, with 32/64/128/256 KB of flash memory, respectively. The CC2530 has various operating modes, making it highly suited for systems where ultralow power consumption is required. Short transition times between operating modes further ensure low energy consumption ${ }^{[8]}$. 


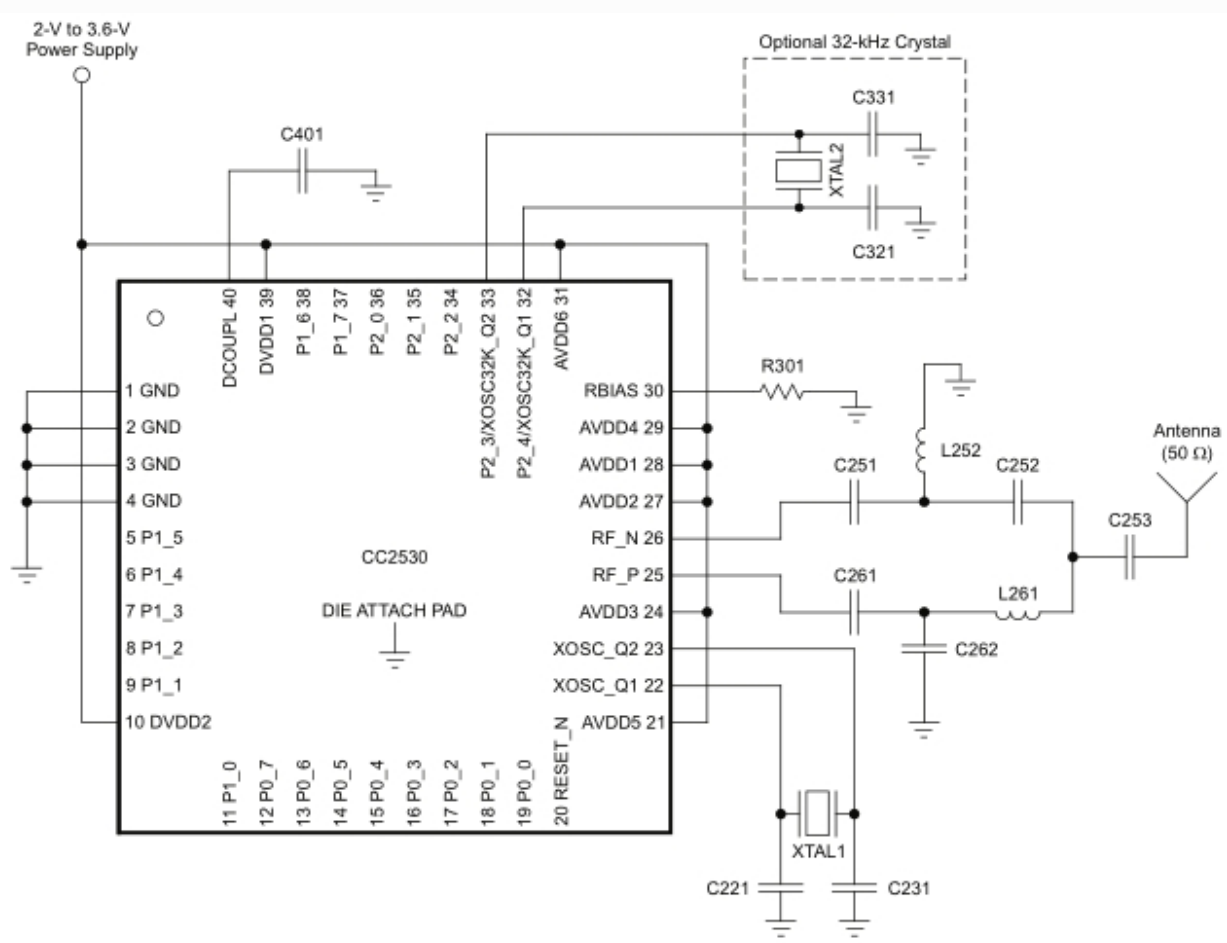

Fig.3 CC2530 Application Circuit

\section{Software design}

Video acquisition module software design, The software flow chart of video capture module is shown in Fig4. The video acquisition procedure is shown as below: First, open the video acquisition device, open the corresponding device file, then set the equipment parameter. The system need to check whether the device is a legitimate video capture device, and to acquire the capabilities of the device. When the memory space for the device has been allocated, image acquisition, data storage and processing are performed. After that, the process ends, the video image acquisition is terminated, the memory is released, and the camera is turned off ${ }^{[9]}$.

Software design of the face detection module, The software design flow chart of the face detection module is shown in Fig4. In the face detection process, Video capture device initialization and buffer initialization are completed first, and then the system judges whether the image data meets the requirement. If the result meets the criteria, the detection device is turned on to start face detection. When the detection results are read, close the testing equipment and video capture equipment, then the video capture process ends ${ }^{[10]}$. 


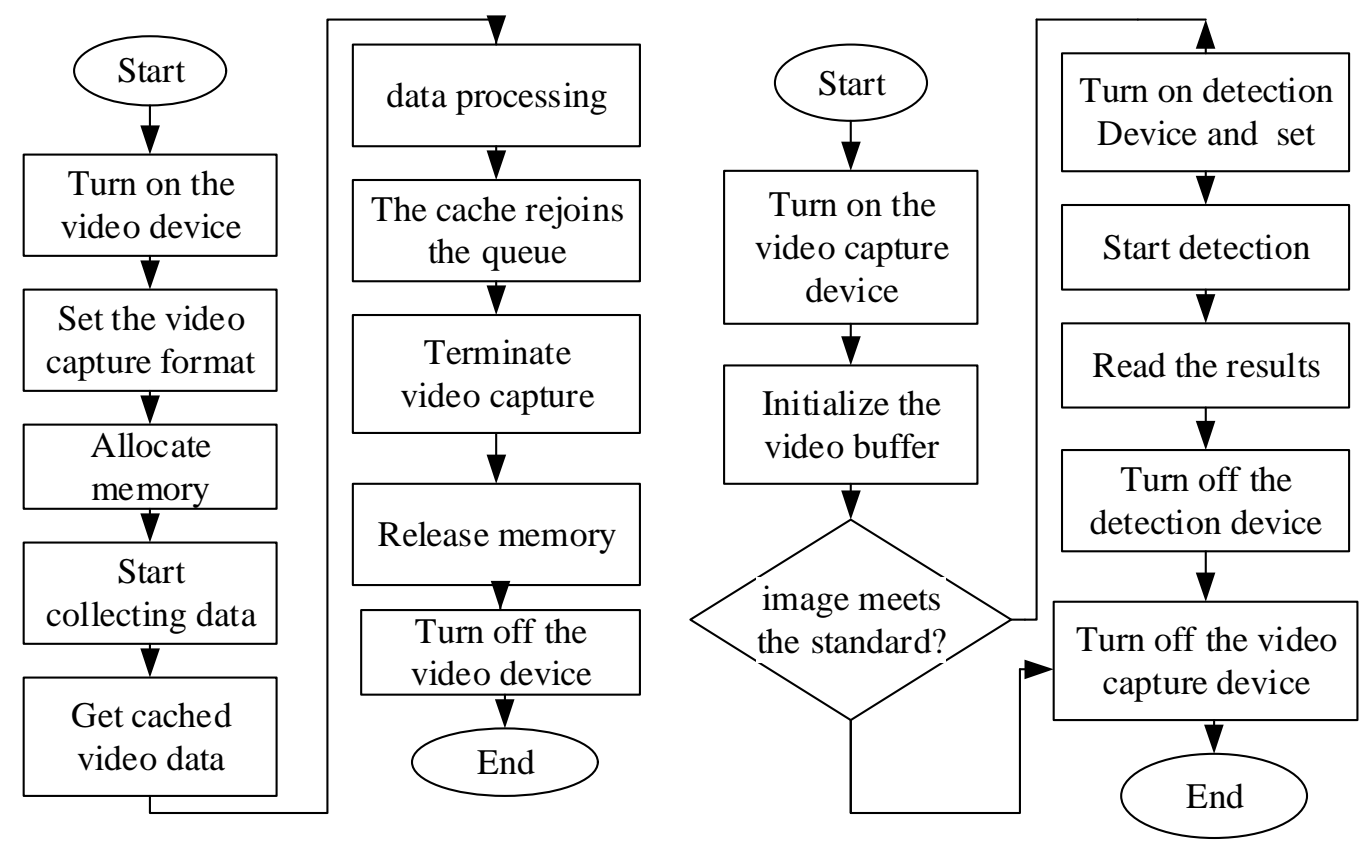

Fig.4 Video acquisition software flow chart and the software design flow chart of the face detection module

\section{Conclusion}

Based on ZigBee technology, a face character recognition intelligent monitoring system is designed, multi-target facial features detection is implemented, the face detection result is accurate, and the output image are very clear. During the running time of the system, the testers' facial activity is smooth, and the system can continuously track the face image within the effective monitoring range. 22490 frames are captured, and 22,271 frames are successfully detected, the detection success rate is $99 \%$ in the test. The test results show that the system is a reliable system which can monitor the surrounding environment in real time. Improving the detection success rate of the system is the focus of follow-up work.

\section{Acknowledgement}

This work is supported by the Natural Science Foundation of Shaanxi Province（2016JK2038）.

\section{References:}

[1] Qin Lin-lin, Design of Greenhouse Intelligent Monitoring System Based on Internet of Things ,Transactions of the Chinese Society for Agricultural Machinery(2014)

[2] Yu Hai-bin, Research and Realization of Moving Target Detection and Tracking Algorithm Based on Intelligent Monitoring System, Research on Intelligent Monitoring System of Clubhouse Based on Face Recognition (2013)

[3] Ruan Lin-lin, Research on Intelligent Monitoring System of Clubhouse Based on Face Recognition, Nanjing University of Science \&Technology(2012)

[4] Wu Qun, A Summary of Modern Intelligent Video Surveillance, Application Research of Computers(2015)

[5] He Shi-wei, Research and Application of Intelligent Monitoring and Alarming System, Donghua University(2016)

[6] Dong Hao-ming, Research on Intelligent Monitoring System of Rice Seedling Environment 
Based on ZigBee Network, Heilongjiang Bayi Agricultural University(2016)

[7] Andrea Pennisi, Online real-time crowd behavior detection in video sequences, Computer Vision and Image Understanding(2016)

[8] Hongrui Cao, Xingwu Zhang, The concept and progress of intelligent spindles: A review, International Journal of Machine Tools and Manufacture (2017)

[9] Omolbanin Yazdanbakhsh, An Intelligent System for Livestock Disease Surveillance, Science of the Total Environment (2016)

[10] Hsueh-Hsien Chang, Non-intrusive fault identification of power distribution systems in intelligent buildings based on power-spectrum-based wavelet transform, Energy \& Buildings (2016) 